\title{
The Role of Nurses in Diabetes Care: A Qualitative Study
}

\author{
Muna Alshammari1, Richard Windle², Dianne Bowskill², Gary Adams² \\ ${ }^{1}$ College of Nursing, Public Authority for Applied Education and Training, Shuwaikh Educational Complex, Kuwait \\ ${ }^{2}$ Faculty of Medicine \& Health Sciences, University of Nottingham, Nottingham, UK \\ Email: alshammari_pinky@msn.com,Richard.windle@nottingham.ac.uk,Dianne.bowskill@nottingham.ac.uk, \\ gary.adams@nottingham.ac.uk
}

How to cite this paper: Alshammari, M., Windle, R., Bowskill, D. and Adams, G. (2021) The Role of Nurses in Diabetes Care: A Qualitative Study. Open Journal of Nursing, 11, 682-695.

https://doi.org/10.4236/ojn.2021.118058

Received: June 24, 2021

Accepted: August 20, 2021

Published: August 23, 2021

Copyright (๑) 2021 by author(s) and Scientific Research Publishing Inc. This work is licensed under the Creative Commons Attribution International License (CC BY 4.0).

http://creativecommons.org/licenses/by/4.0/ (c) (i) Open Access

\begin{abstract}
Purpose: Nurses are key players in the care of diabetes patients. It is not clear how nurses experience the diabetes care role within a multidisciplinary care context. This article reports the perspectives of nurses working in one $\mathrm{Na}$ tional Health Service trust in the United Kingdom, about their role in diabetes care. Methods: The study employed a descriptive qualitative approach. Participants were nurses who worked in two diabetes clinics in one National Health Service trust in the United Kingdom. The study included 10 female participants, who provided nursing care to patients with diabetes. Data were collected between May and September 2017. Results: Three themes were generated in relation to the role of nurse in diabetes care: 1) Nurses' performed role: the role performed by nurses in diabetes care was regarded as complex and one that required appropriate preparation and support; 2) Implications of the nurses' performance: nurses engaged in multiple activities that aimed at providing holistic and patient centred care, and 3) Challenges facing nurses in diabetes care: nurses reported facing significant challenges that interfered with their care role and which could negatively affect patient outcomes. Conclusions: Nurses play a critical role in the care of diabetes patients and require significant support to undertake their role.
\end{abstract}

\section{Keywords}

Diabetes, Diabetes Nurse, UK, Care, Role

\section{Introduction}

Diabetes is increasingly becoming a global public health concern with its prevalence steadily on the rise [1]. A study by Saeedi and colleagues Saeedi et al. [2] reported a prevalence of diabetes at $9.3 \%$ in 2019 , which is projected to rise to 
$10.2 \%$ by 2030 and $10.9 \%$ by 2045 . United Kingdom (UK) is reported as one of the Western European countries with the highest number of people with Diabetes with a reported increase in newly diagnosed cases [3]. Type 2 Diabetes (T2D), defined as chronic hyperglycaemia caused by decreased insulin production or impaired insulin function [4] comprises 90 percent of people who are affected by diabetes globally [1]. Because it may show signs of disease after a long time of acquisition, managing T2D is an on-going challenge in the healthcare sector, as its complications impacts health outcomes and healthcare costs [1].

Managing diabetes requires new models of care and practice. Increasingly, healthcare systems are adopting nurse-led models that are believed to be patient centred, as opposed to the more traditional physician-led model that focuses on medical treatment and cure. Empowering nurses with more independent roles in diabetes care has been suggested as a possible initiative to improve the outcomes of patients with diabetes [5]. When comparing a nurse-led model with traditional care, Li and colleagues [6] demonstrated that the nurses led model was more effective in improving glycemic control and reducing diabetes distress.

In practice, the management of diabetes in the UK involves a multidisciplinary approach, with an integration of different care providers such as physicians, nurses [7] and other professionals such as dieticians and podiatrists [8] [9]. A multidisciplinary approach is believed to improve the quality of diabetes care compared with the individual patient-provider care model [1] [7]. We sought to explore how nurses in one NHS trust working in diabetes care undertook their role and how their role was influenced by other colleagues, being part of a multidisciplinary team. The article is part of a larger $\mathrm{PhD}$ study undertaken by the lead author at a UK university. The lead author has expertise in chronic disease and qualitative research. The article will provide evidence that could contribute to the understanding of the role provided by nurses to diabetes patients and how nurses can be supported to work in a team to contribute to improvement in the care provided to diabetes patients.

\section{Methods}

\subsection{Study Design}

The current study employed a descriptive qualitative approach, to explore the nursing care offered in two diabetes clinics in one NHS trust in the UK.

\subsection{Sample/Participants}

Sampling aimed at selecting samples that would exhibit relevant descriptions and behavioural actions applicable to the study's aims and objectives. A combination of both convenience and purposive sampling was used to select participants who directly worked with diabetes patients and were available to participate in the in-depth interviews. We aimed to include a total of 15 participants, however, consistent with qualitative research, the principle of data saturation 
was employed in determining the final sample size. Participants were labelled with code numbers followed by the site code (NUH).

\subsection{Study Settings}

Two sites from one NHS hospital in the UK were identified as research sites for the study. Both sites were providing comprehensive care for patients with diabetes in hospital based outpatient clinics. In each site, one diabetes clinic was included.

\subsection{Data Collection}

Following ethical approvals, the clinics' managers arranged for the researcher to meet with staff, where she introduced herself and made known her intentions. Following provision of relevant information, potential participants were requested to consent for study participation which included face-to-face interviews and observations. Consent to observe a care session was also sought from patients and other healthcare providers who would be involved during such a session.

Semi-structured interviews were conducted with diabetes care nurses using a semi-structured interview guide (Appendix 1). Participants were interviewed in a private room at each clinic. Following consent of participants, interviews were recorded using a digital voice recorder (Philips 1100 recorders). Hand-written notes were taken and reflective accounts of interviews were documented soon after each interview. Employing observations in this study enabled the researcher to observe the nature of interactions between nurses and patients during consultations, allowing the researcher to explore the actual roles that nurses were undertaking on a day-today basis within the clinics. A convenient place to sit was selected that allowed the researcher to observe and take notes basing on facial expressions and body language of both nurses and patients. We drew on questions generated from the observations to enrich the interview schedules and improve the trustworthiness of the data. During observations of nursing activities, field notes were written, but the activities were not audio or tape-recorded. Participants' names were not used in the field notes. The study observations covered five hours in the morning and three hours in afternoon. Data was collected between May and September, 2017.

\subsection{Ethical Considerations}

The study was part of a $\mathrm{PhD}$ program and as such the protocol was approved by the PhD students' University and the National Institute of Health. In addition, formal permission to undertake the study in the clinics concerned was sought and granted.

\subsection{Confidentiality and Protection of Participants}

All information given by the participants was held in the strictest confidence, and only the researcher with the supervisory team had access to it. All digital 
recordings and computer-based copies of the transcripts were stored in a password-protected computer on the university database server and treated according to the university guidelines and data protection laws. All participants' hard copy forms will be kept in a lock-protected cupboard at the university for seven years from the end of the study.

\subsection{Data Analysis}

Each interview was transcribed verbatim immediately after the interview ended, to enable the researcher capture fresh memories of the interview proceedings including emotions and facial expressions. All interviews were conducted in the English language. Transcribing each interview took between five to ten hours. Data analysis was influenced by both the eight steps and six steps suggested by Stake [10] and Miles and Huberman [11] respectively, while the overall scene was guided by Braun and Clarke [12]. For each data set, we followed general strategies which include: 1) preparing and organising the data, 2) reducing the data into themes through a coding process and condensing codes, and 3) presenting the data into figures, tables or a discussion by each source of data collection [13] (p. 180).

The analysis process started with a close reading of the raw text to enable familiarisation and understanding of the content. Notes of thoughts and memos were recorded throughout the analysis process. The initial stage of analysis used an inductive thematic analysis approach, where codes were generated from the data. Initial coding of each transcript was made separately and all codes were labelled and provided with descriptions. "Freenode" and "tree node" tasks were employed to arrange data in the computer and to sort and rank codes, which enabled the development of a hierarchy of codes. The coding process stopped when no dissimilar codes emerged, when refinements were not adding any new codes, and when the study's objectives had been met. A qualitative computer analysis programme (QSR NVivo10) was used to sort codes and categorise data for detailed analysis.

\subsection{Maintaining Rigour}

To maintain rigour, various individuals were involved in the coding process including the principle researcher and the $\mathrm{PhD}$ supervisors. The first author's previous work and research experience in chronic diseases and medical-surgical nursing could have influenced her interpretation of the data and she ensured that this background was considered throughout the research process. Additionally, the current study used multiple data sources that included in-depth individual interviews and observations (triangulation) that allowed for cross-data validity checks, hence strengthening the credibility of the research.

\section{Findings}

This article reports findings about the role of nurses in providing diabetes care in one selected NHS trust in the UK. The study included 10 female participants, 
who were between the age of 30 to 66 years, with a nursing experience ranging from four to 38 years. The nurses' qualifications ranged from Nursing Diploma to Master's degree and their years of experience in diabetes clinical care ranged from two to 21 years, with the majority having more than 10 years' experience in diabetes care. All participants approached to take part in the study participated.

The data from individual interviews and participant observations generated three key themes, pertaining to their role in diabetes care. These included: Nurses' performed role, implications of nurses' performance, and challenges facing nurses in diabetes care. These findings are summarised below with representative quotes to support the interpretations made by the researchers.

\subsection{The Performed Role of a Nurse in Diabetes Care}

The performed role of nurses in diabetes care was found to be diverse and challenging. Nurses reported participating in various activities such as health education and promotion, providing patient support, prescribing, and other interprofessional roles. Although most of these roles pertained to those of a general nurse, providing them in a diabetes care setting came with special responsibilities. The performed role of nurses in diabetes care was further presented under several sub-themes.

\subsubsection{Health Education and Health Promotion}

Nurses reported providing health promotion messages that they felt were helpful for the patients. They taught patients how to interpret laboratory results, and spent much time in consultation with patients, gathering baseline information about them, and providing relevant information about diabetes care. Patient empowerment and encouragement for self-management was included in diabetes care and management.

"We ask the patients what they want to get out of the consultation and then I personally have a duty of care to make sure that they have the correct information. So, I will deliver education in a way that I feel is appropriate to the patient." (Nurse 2-NUH)

"We provide professional education. We do provide that on either an individual basis or group educational sessions, as we noticed that some patients are better in understanding and appropriately controlling their glycaemic level in group sessions." (Nurse 8-NUH)

The main barriers to providing health education included patient's demand for focused and detailed information about their conditions, their progression, and treatment. Nurses also reported language barrier, which they overcame by using drawings (with non-English speakers). Such processes sometimes required additional resources.

The patient cannot speak or understand English very well. The nurse was trying to understand what he was saying and to make sure he understands what she is saying. Alternatively, she was drawing for him and writing to make sure he understood what she said. The consultation took more than 30 minutes, but the 
patient looked comfortable and appeared to indicate understanding of what the DN said. (Observation 6-NUH)

\subsubsection{Providing Holistic Care}

Nurses provided various kinds of support to their patients to respond to their individual needs. They reported approaching their patients holistically, by attending to other needs that are not directly related to diabetes. One nurse reported how her unit took care of patients' diverse needs including cultural and financial issues, foot care, physical and psychological care, as well as social support:

"Our patients come with different needs and we make sure we cover these needs. Some of them [patients] have needs that are related to their cultural backgrounds, others have financial problems and in that case, we try to provide some solutions, though this needs other interventions that are not under our scope of practice..." (Nurse 2-NUH)

Providing care that focused on the individual patients' needs was reported to increase the quality of care provided to T2D patients and was perceived to result in better patient outcomes such as successful diabetes control and patient satisfaction with care.

The effort $1 \mathrm{~m}$ providing here and patients appreciate each time they come to the clinic." (Nurse 7-NUH)

\subsubsection{Prescribing}

While nurses undertook an important role in providing patients with medication advice. Nurses also reported being able to prescribe after successfully completing a prescribing course. The prescription role generally included prescribing/adjusting insulin dosages.

"I provide consultations to patients, prescribe or adjust his insulin, and check his routine readings and his diary to make sure he is following the treatment plan." (Nurse 3-NUH)

" $\mathrm{m}$ a nurse prescriber. I can prescribe insulin and I can change the insulin, and mix insulin ..." (Nurse 8-NUH)

Many nurses felt it is important to be authorised to prescribe treatments beyond diabetes, as diabetes can contribute to other conditions. For instance, a nurse reported that her patient came with high cholesterol levels and needed medication such as statins, and despite her feeling competent to prescribe the medication, she was not authorised to do so in her current setting.

"My patient this morning is actually having high cholesterol levels and I know the statin works and he is tolerable but I $m$ not able to prescribe it..." (Nurse 5-NUH)

"Actually, I cannot prescribe other than for diabetes as this is a medic's role." (Nurse 6-NUH)

While taking on the prescriber role may add workload to the nurse working in diabetes care, many viewed it as an added resource to quality care provision and in some cases time saving. For example, waiting for doctors to prescribe would 
delay a patient's treatment and could lead to clinic delays in general.

"Some patients urgently need some treatment and we should refer them ... That can delay the start of treatment ..., prescribing makes the flow of the clinic quicker..., it saves a patient's time and their experience would become better as they can start taking that medication right away." (Nurse 10-NUH)

\subsubsection{Interprofessional Roles}

Nurses working in diabetes care were undertaking roles in collaboration with other nurses and other healthcare professionals. The roles undertaken on patients other than diabetes patients made the nurse working in diabetes care added more workload to them and were sometimes viewed as disruptive to the diabetes care role.

" $A$ nurse or a doctor at the ward calls me up to teach a non-diabetic patient [...] Those things might look small but they are interrupting our work." (Nurse $1-\mathrm{NUH})$.

On some occasions, it was reported that the nurses' role interpretations/interruptions by other colleagues raised the issue of power relations, especially within the nurse/doctor relationship. Some nurses, who took on additional/multiple roles outside their prescribed scope of work felt it being unfair to them as they seemed to be doing someone else's job.

"I will not have a registrar so I will be my own registrar ... It is about adding more pressure and more responsibilities that are more than what you could handle. On the wards, as well I do more work. We [nurses] have to make the right decision about patient discharge and this can sometimes be very challenging." (Nurse 9-NUH)

Most times, undertaking additional roles was found challenging and sometimes compromising the quality of care provided to diabetes patients.

"Doing work more than what you should do is a conflict. Sometimes we do not have a doctor available and we are seeing patients on the wards and it is a tricky situation." (Nurse 8-NUH).

\subsection{Implications of the Nurses' Role in Diabetes Care}

Nurses discussed how they felt about the contribution of their role to the overall care of diabetes patients. They felt their role contributed to providing timely interventions that are patient focused and that resulted into better quality of life for the patients. Their role was perceived as one that provided patient-centred care, enhanced their autonomy in practice, and expanded their scope of practice.

\subsubsection{Providing Patient-Centred Care}

The nurses regarded the care provided to diabetic patients as patient-centred, as it aimed at addressing the holistic care needs of their patients. Nurses admitted that on some occasions they were not able to personally resolve patients' concerns; however, they provided pathways for them to follow so as to address their worries and concerns. 
"... the care we provide is holistic, we cover patients' needs holistically." (Nurse $7-N U H)$

"I certainly have patients who would say to me I don't need to see a doctor today because I $m$ happy with what we've done". (Nurse 2-NUH)

Nurses reported that a patient-centred approach facilitated a two-way communication, where the nurse understands and covers the patient's needs, which can sometimes be complex. This dedicated communication could lead to a trusting relationship between the nurse and the patient and resulted into treatment adherence among patients. A patient centred approach was viewed as holistic, empowering, and one likely to positively impact on patients' outcomes.

“... we don't consider our patients as only patients; we know a lot about their lives. We [nurses] support people ... This is indicated by patients following up with their treatment plan." (Nurse 8-NUH)

\subsubsection{Enhancing Professional Autonomy}

Nurses reported that the diabetes care role shaped their self-confidence in managing their patients. Expression of self-confidence can be considered as an enabler for the nurses' abilities to make decisions, solve problems, and plan patient care independently. Nurses working in diabetes care had their own patients, where each of them was dedicated to take care of particular clients, which ensured patients' continuity of care. The nurses had their own patient caseloads and developed ways of working to deal with most patients' issues, relating both to diabetes care and non-diabetes issues. Nurses who reported capability to adjust patients' insulin intake when required felt that their professional autonomy was enhanced.

\subsubsection{Professional Development-Extending the Role}

The extended role was explained by diabetes nurses as their ability to prescribe both diabetes and non-diabetes medication, in order to facilitate holistic patient care. Nurses mentioned drawbacks to this function such as regarding prescribing as part of a doctor's role while some viewed it as an unsafe procedure if specific capacity building is not provided.

"So, you would not do anything you are not qualified to do. But there is always an expectation that you can have extra patients put into your schedule." (Nurse 2-NUH)

Nurses considered the provision of diabetes care an on-going practice and meetings with their peers and doctors at the clinic contributed to keeping their knowledge about new treatments in diabetes up-to-date. Developing the role of a diabetes nurse by extending their role and keeping up-to-date with new treatments and practice was thought to contribute to enhancing patient care.

\subsection{Challenges Faced by Diabetes Nurses While Undertaking Their Care Role}

Nurses reported various external forces that interfered with the nurses' work while providing diabetes care and thus affected its quality. These issues included 
staff shortages and heavy workload, remuneration concerns and negative relationships with other staff members as elaborated below.

\subsubsection{Shortage of Staff}

Nurses expressed that the limitations in staff numbers, including both doctors and nurses required them to work more than their normal workload. This affected the quality of their work and also impacted their own health. The nurses identified a number of issues that could arise as a result of long hours of work and exhaustion.

"We are expected to do more and more and see more and more people as there is a lack of staff. You know there are so many things to be done by one person and that makes us feel tired and exhausted." (Nurse 6-NUH)

The wellbeing of nurses is a fundamental factor in patient care. If the nurse is exhausted and frustrated, she/he may lose her sense of caring as well as being prone to making mistakes, putting patients at risk.

\subsubsection{Increased Workload}

Apart from their few numbers, nurses considered that they were expected to undertake more roles than was within their workload. It was evident that the nurses did not have sufficient time to fully manage all the aspects of patient care, a problem which impacted on their ability to provide quality patient care.

“... there is no limit to the expectation that we would do. There is your seniors' expectation that you discharge duties within the scope of your profession. So, you would not do anything you are not qualified to do. But there is always an expectation that you can fit extra patients into your schedule." (Nurse 2-NUH)

As a result of the nurses' shortages and the continuing pressure of work, they considered that they were expected to undertake more than they could reasonably do on a daily basis, which was frustrating to them.

\subsubsection{Lack of Recognition and Support}

Organisational dynamics was reported to sometimes hinder the nurses' performance. Nurses reported that conflicting expectations from senior management increased their workload and time pressure.

"The only time I get upset is when P ve got a full workload and you get the nurse in charge saying to me, you have to go and see this patient. I can't go and see this patient as I have another patient. However, she [the in-charge nurse] said you' ve got to go." (Nurse 2-NUH)

Nurses also reported interruptions during their work caused by fellow staff such as doctors and other nurses which interfered with their completion of tasks. Occasionally, nurses reported being contacted to accomplish tasks that were not their responsibility.

"We have not got a doctor here at all and the next couple of days $1 \mathrm{~m}$ going to be on my own here..." (Nurse 9-NUH) 


\subsubsection{Financial Conflict}

A few nurses expressed concerns about not receiving an appropriate level of income to match their role. Some complained that their income was just the same as for nurses working in general departments which are non-specialised, while others felt they did more work compared with the general nurses who are sometimes paid more than them. This was a demotivating factor for the nurses.

"We are not having enough income compared to what we are doing and what we receive is similar to other nurses in other departments. We are covering quiet huge parts in diabetes care... We just cannot ignore the patients when they come seeking help." (Nurse 10-NUH)

\section{Discussion}

The current study reported the perspectives of nurses from a selected NHS trust, about their role in providing care to diabetes patients. Nurses reported engaging in various activities that could exceed their job description or scope of work. For the provision of quality care, it is essential for the role of nurses to be guided and defined [14].

Nurses in the current study reported adopting an individualised approach to care, in which each patient's needs are handled on a personal basis. Although this approach can be demanding, it has been found to result into better patient outcomes [6], and should be promoted in diabetes care practice. To provide individualised care, nurses engaged into multiple activities aimed at meeting the various care needs of their patients, of which some form the general nurses' role in the UK [15] and some were beyond diabetes care. A holistic approach to care is considered patient-centred and has been associated with improved patient health outcomes. In addition, providing holistic care means that the nurses get to spend more time with their patients which has been reported to result into patient satisfaction [16]. This approach could also be useful in minimising human resources and contribute to saving costs. These data support the need for adopting a holistic approach to patient care, while further research will be essential to highlight how this approach is implemented in practice and its actual impact on diabetes patient health outcomes.

Providing holistic care may also mean that nurses perform more roles including those outside diabetes care. Performing additional roles was perceived in the current study as a move towards professional autonomy, where nurses wished to have the authority to attend to their patients holistically, including prescribing medications. Consistent with literature that indicates that nurses in the UK have moved towards performing advanced roles [17], nurses in the current study reported engaging in advanced roles such as prescribing, which they highly supported and recommended. It has been documented that nurse prescribing is a successful practice that supports a range of services such as holistic management of chronic diseases, including diabetes [18]. Research undertaken in diabetes care has also supported this initiative indicating that it improves the quality of care provided by enhancing timely access to medications [19] [20]. However, to 
advance their role, it is essential that nurses are equipped with adequate knowledge about the disease condition and its treatment as nurses' poor consultation skills have been attributed to poor patient outcomes [21]. This suggests further training for nurses working with diabetes patients.

To improve the care of patients, it is suggested that nurses work in a team with other healthcare providers, to ensure the integration and coordination of patient care [1] [7]. In the current study, it was suggested that nurses could not appropriately provide quality care to the patients alone and thus required other members of the health care team. A team-based approach was shown to further strengthen the provision of patient-centred care. Although this approach could be helpful, it was reported in the current study to result into work overload, when nurses undertook roles supposed to be done by other care providers. This challenge could also be associated with shortage of manpower, which is an outstanding challenge in the provision of diabetes care [22].

Nurses in the current study further reported being demotivated by the lack of adequate resources such as private space which affected the quality of care provided, while some felt demotivated due to underpayment. Whilst not measured in the current study, nurse retention is influenced by factors that affect motivation such as support from colleagues and superiors, and empowerment [23]. Not meeting the needs of nurses has also been found to contribute to poor work outcomes [24], while addressing them is associated with quality care and healthcare providers' satisfaction [25]. A supportive environment is advocated for in healthcare facilities to enhance the role of nurses in $\mathrm{T} 2 \mathrm{D}$ care.

\section{Limitations}

Being a single institution study, we recognize that our findings may not be applicable beyond the context of the study, hence the need to undertake similar studies in other diabetes care settings in the UK and internationally, to generate more representative conclusions that could inform strategies to improve the care of diabetes patients. Furthermore, our study employed a purely qualitative approach. While we recognize that qualitative studies can be in themselves pragmatic, they stand a limitation of self-reporting bias. Hence, the application of recommendations in this study should be made in consideration of other related studies.

\section{Conclusion}

The current study aimed to establish the role of nurses in diabetes care in a selected NHS trust in the UK. The findings indicate that the role performed by nurses in diabetes care may be complex and requires appropriate preparation and support. Nurses in the current study engaged (or would want to engage) in multiple activities that aimed at providing holistic and patient centred care. In performing their care role, nurses encountered significant challenges that could interfere with their care role and was feared to affect patient outcomes. Nurses 
also reported feeling inadequately prepared for the advanced roles in diabetes care, and therefore required additional capacity building to facilitate their role. Support of management and other healthcare providers may be critical in enabling nurses working with diabetes patients to undertake their role. This study should be extended to more NHS trusts to provide more generalizable evidence about the role of nurses in diabetes care in the UK.

\section{Conflicts of Interest}

The authors declare no conflicts of interest regarding the publication of this paper.

\section{References}

[1] American Diabetes Association (ADA) (2020) 4. Comprehensive Medical Evaluation and Assessment of Comorbidities: Standards of Medical Care in Diabetes2020. Diabetes Care, 43, S37-S47. https://doi.org/10.2337/dc20-S004

[2] Saeedi, P., Petersohn, I., Salpea, P., Malanda, B., Karuranga, S., Unwin, N., et al. (2019) Global and Regional Diabetes Prevalence Estimates for 2019 and Projections for 2030 and 2045: Results from the International Diabetes Federation Diabetes Atlas, 9th Edition. Diabetes Research and Clinical Practice, 157, Article ID: 107843. https://doi.org/10.1016/j.diabres.2019.107843

[3] World Health Organization (WHO) (2016) Global Report on Diabetes. World Health Organization, Geneva.

[4] World Health Organization (WHO) (2006) Diagnosis of Diabetes Mellitus and Intermediate Hyperglycemia: Report of a WHO/IDF Consultation. World Health Organization, Geneva, 50.

[5] Juul, L., Maindal, H.T., Frydenberg, M., Kristensen, J.K. and Sandbaek, A. (2012) Quality of Type 2 Diabetes Management in General Practice Is Associated with Involvement of General Practice Nurses. Primary Care Diabetes, 6, 221-228. https://doi.org/10.1016/j.pcd.2012.04.001

[6] Li, D., Elliott, T., Klein, G., Ur, E. and Tang, T.S. (2017) Diabetes Nurse Case Management in a Canadian Tertiary Care Setting: Results of a Randomized Controlled Trial. Canadian Journal of Diabetes, 41, 297-304.

https://doi.org/10.1016/j.jcjd.2016.10.012

[7] King, O., Nancarrow, S., Grace, S. and Borthwick, A. (2017) Diabetes Educator Role Boundaries in Australia: A Documentary Analysis. Journal of Foot and Ankle Research, 10, Article No. 28. https://doi.org/10.1186/s13047-017-0210-9

[8] Diabetes UK (2013) Diabetes Prevalence. http://diabetes.org.ik/professionals/position-statements-reports/statistics

[9] National Institute for Clinical Excellence (NICE) (2008) Type 2 Diabetes: National Clinical Guideline for Management in Primary and Secondary Care London. The Royal College of Physicians, UK. https://www.ncbi.nlm.nih.gov/books/NBK53885/

[10] Stake, R.E. (1995) The Art of Case Study Research. Sage Publications, Thousand Oaks.

[11] Miles, M.B. and Huberman, A.M. (1994) Qualitative Data Analysis: An Expanded Sourcebook. Sage Publications, Thousand Oaks.

[12] Braun, V. and Clarke, V. (2006) Using Thematic Analysis in Psychology. Qualitative Research in Psychology, 3, 77-101. https://doi.org/10.1191/1478088706qp063oa

[13] Creswell, J.W. (2012) Qualitative Inquiry and Research Design: Choosing among 
Five Approaches. Sage Publications, Thousand Oaks.

[14] Nikitara, M., Constantinou, C.S., Andreou, E. and Diomidous, M. (2019) The Role of Nurses and the Facilitators and Barriers in Diabetes Care: A Mixed Methods Systematic Literature Review. Behavioral Sciences, 9, Article No. 61. https://doi.org/10.3390/bs9060061

[15] Beck, J., Greenwood, D.A., Blanton, L., Bollinger, S.T., Butcher, M.K., Condon, J.E., et al. (2017) 2017 National Standards for Diabetes Self-Management Education and Support. Diabetes Care, 40, 1409-1419. https://doi.org/10.2337/dci17-0025

[16] Doran, D., Harrison, M.B., Laschinger, H., Hirdes, J., Rukholm, E., Sidani, S., et al. (2006) Relationship between Nursing Interventions and Outcome Achievement in Acute Care Settings. Research in Nursing \& Health, 29, 61-70. https://doi.org/10.1002/nur.20110

[17] Courtenay, M., Carey, N. and Burke, J. (2007) Independent Extended and Supplementary Nurse Prescribing Practice in the UK: A National Questionnaire Survey. International Journal of Nursing Studies, 44, 1093-1101. https://doi.org/10.1016/j.ijnurstu.2006.04.005

[18] Courtenay, M., Stenner, K. and Carey, N. (2010) The Views of Patients with Diabetes about Nurse Prescribing. Diabetic Medicine, 27, 1049-1054.

https://doi.org/10.1111/j.1464-5491.2010.03051.x

[19] Carey, N., Stenner, K. and Courtenay, M. (2010) Stakeholder Views on the Impact of Nurse Prescribing on Dermatology Services. Journal of Clinical Nursing, 19, 498-506. https://doi.org/10.1111/j.1365-2702.2009.02874.x

[20] Stenner, K. L., Courtenay, M. and Carey, N. (2011) Consultations between Nurse Prescribers and Patients with Diabetes in Primary Care: A Qualitative Study of Patient Views. International Journal of Nursing Studies, 48, 37-46. https://doi.org/10.1016/j.ijnurstu.2010.06.006

[21] Jansink, R., Braspenning, J., van der Weijden, T., Elwyn, G. and Grol, R. (2010) Primary Care Nurses Struggle with Lifestyle Counseling in Diabetes Care: A Qualitative Analysis. BMC Family Practice, 11, Article No. 41. https://doi.org/10.1186/1471-2296-11-41

[22] Burtson, P.L. and Stichler, J.F. (2010) Nursing Work Environment and Nurse Caring: Relationship among Motivational Factors. Journal of Advanced Nursing, 66, 1819-1831. https://doi.org/10.1111/j.1365-2648.2010.05336.x

[23] Laschinger, L.H.K. (2008) Effect of Empowerment on Professional Practice Environments, Work Satisfaction, and Patient Care Quality: Further Testing the Nursing Worklife Model. Journal of Nursing Care Quality, 23, 322-330. https://doi.org/10.1097/01.NCQ.0000318028.67910.6b

[24] Aiken, L.H., Cimiotti, J.P., Sloane, D.M., Smith, H.L., Flynn, L. and Neff, D.F. (2011) Effects of Nurse Staffing and Nurse Education on Patient Deaths in Hospitals with Different Nurse Work Environments. Medical Care, 49, 1047-1053. https://doi.org/10.1097/MLR.0b013e3182330b6e

[25] Amaral, A., Ferreira, P., Cardoso, M. and Vidinha, T. (2014) Implementation of the Nursing Role Effectiveness Model. International Journal of Caring Sciences, 7, 757770 . 


\section{Appendix 1: Semi-Structured Interview Guide for the Study}

1) What do you do as a nurse in this clinic?

2) Could you tell me about your role in providing health promotion?

3) What is your involvement in medication?

4) What guidelines and protocols guide your work?

5) How independent do you work? 